than has been done before and is applied to transversal deformations and vibrations of plates and to the general 2-dimensional problem of elasticity. The chapter properly concludes with Plateau's problem which has recently entered upon a new phase through J. Douglas' ideas, and to which Courant himself has contributed largely during his last New York years.

The present volume is sprinkled throughout with a wealth of little new illuminating observations which this review had to skip. The author apologizes that lack of time prevented him from fitting out his book with a full sized index of literature and such paraphernalia. The same reason may be responsible for quite a few misprints on which the reader will occasionally stumble. But perhaps even these minor faults deserve praise rather than blame. Although I know that a craftsman's pride should be in having his work as perfect and shipshape as possible, even in the most minute and inessential details, I sometimes wonder whether we do not lavish on the dressingup of a book too much time that would better go into more important things.

HermanN Weyl

\title{
CONTRIBUTIONS TO THE CALCULUS OF VARIATIONS
}

Contributions to the Calculus of Variations, 1933-1937. (Theses submitted to the Department of Mathematics at the University of Chicago.) University of Chicago Press, 1937. 7+566 pp.

This volume is the third in the series of contributions to the calculus of variations published by the Department of Mathematics of the University of Chicago since 1930.* It contains thirteen doctoral dissertations, written under the direction of Professors Bliss, Graves, and Reid. The reader acquainted with the work of these mathematicians can make a fair guess as to the way in which responsibility for these papers is to be distributed among them.

The book is uniform in appearance and in style with the earlier ones. About half the papers are followed by a list of references or a bibliography. They are all written with care, conditions are carefully stated, and conclusions are clearly set out. Their contents give a clear picture of the direction in which the work in the calculus of variations has been developed at the University of Chicago in recent years. The contributions contained in the volume are the following:

1. The problem of Lagrange with finite side conditions, by J. W. Bower (pp. 1-52).

2. Fields for multiple integrals in the calculus of variations, by Byron Cosby, II (pp. 53-84).

3. The minimum of a definite integral with respect to unilateral variations, by J. D. Mancill (pp. 85-164).

4. The Hamilton-Jacobi theory for the problem of Lagrange in parametric form, by Van Bauman Teach $\dagger$ (pp. 165-206).

5. Sufficient conditions for a minimum in the problem of Lagrange with isoperimetric conditions, by I. E. Perlin (pp. 207-242).

6. A boundary value problem of the calculus of variations, by E. P. Wiggin (pp. 243-276).

* The first of these was reviewed in this Bulletin, vol. 38 (1932), p. 617; the second in vol. 39 (1933), p. 641.

$\dagger$ Deceased before the publication of the volume. 
7. The inequalities of Morse for a parametric problem of the calculus of variations, by R. H. Bardell (pp. 277-312).

8. Conditions for a minimum of a functional, by H. H. Goldstine (pp. 313-358).

9. Studies of the inverse problem of the calculus of variations, by N. A. Moscovitch (pp. 359-402).

10. The problem of Lagrange with differential inequalities as added side conditions, by F. A. Valentine (pp. 403-448).

11. A generalized form of tice problem of Bolza, by C. H. Denbow (pp. 449-484).

12. The dependence of a focal point upon curvature in the calculus of variations, by

A. S. Householder (pp. 485-526).

13. Discontinuous solutions for the problem of Bolza in parametric form, by M. F. Smiley (pp. 527-566).

The problems with which these papers are concerned do not have very close connection with one another, except insofar as numbers $1,4,5$, and 10 deal with the problem of Lagrange, and numbers 11 and 13 with the problem of Bolza. In the discussion which follows, the papers will be considered in accordance with this grouping.

Miss Bower (pp. 1-52) discusses the problem of minimizing the integral $\int F\left(x, y_{1}, \cdots, y_{n}, y_{1}^{\prime}, \cdots, y_{n}^{\prime}\right) d x$ in the set of arcs $y_{i}=y_{i}(x)$ which are piecewise of class $C^{\prime}$, join two fixed points, and satisfy a system of finite equations $\phi_{\alpha}\left(x, y_{i}\right)=0$, $(\alpha=1, \cdots, m<n)$, for which the matrix $\left\|_{\phi_{\alpha y}}\right\|$ has rank $m$ along these arcs. It is treated by two methods, the first consisting in replacing the finite conditions by differential equations, the second in using the finite equations to eliminate $m$ of the unknown functions. In order to avoid normality difficulties in the first of these formulations, the end conditions are reduced, following Bolza, by leaving the initial values of the functions $y_{1}, \cdots, y_{n}$ undetermined. The problem becomes a Lagrange problem with variable endpoints, to which the usual methods are applicable with some modifications. Necessary conditions and sufficient conditions are thus obtained. In the second part of the paper $n-m$ equations $\phi_{r}\left(x, y_{i}\right)=z_{r}$ are adjoined to the equations $\phi_{\alpha}=0$, so as to obtain a system which is solvable for $y_{1}, \cdots, y_{n}$. The problem is thus transformed into a problem without side conditions, for which familiar methods are available. The paper gives for the first time a complete set of conditions for this problem. It would be interesting to compare the result here found with those which could be obtained by applying the recent work of Hestenes and of Reid in which no assumptions of normality are made.

Paper 4 (pp. 165-206) deals with the Lagrange problem in parametric form for fixed endpoints. By associating with the differential equations $\phi_{\alpha}\left(y, y^{\prime}\right)=0$ an equation $\phi\left(y, y^{\prime}\right)=1$, which defines the parameter, and then treating the problem $\int f\left(y, y^{\prime}\right) d t$ $=\min , \phi_{\alpha}\left(y, y^{\prime}\right)=0, \phi\left(y, y^{\prime}\right)=1$, an ordinary problem in $t y$-space is obtained. For this problem the author develops the usual conditions necessary for a minimum, and also the Hamilton-Jacobi theory. These results are then carried over to obtain corresponding conditions for the parametric problem.

The next paper, by Dr. Perlin (pp. 207-242), is devoted to the theorem of Lindeberg (compare Bolza, Variationsrechnung, p. 515) for the same Lagrange problem $J(C)=\int f\left(x, x^{\prime}\right) d t$, but with isoperimetric side conditions. The class $K$ of admissible curves $x_{i}=x_{i}(t)$ is constituted by continuous rectifiable arcs lying in a bounded closed region of $\left(x_{1}, \cdots, x_{n}\right)$-space which satisfy a set of differential equations $\phi_{\beta}\left(x, x^{\prime}\right)=0$ and a set of isoperimetric conditions $\int_{l_{1}}^{t_{2}} g_{\sigma}\left(x, x^{\prime}\right) d t=l_{\sigma}$. The Weierstrass $E$ function is defined in the usual way in terms of the functions $H \equiv f\left(x, x^{\prime}\right)+\lambda_{\beta} \phi_{\beta}+\mu_{\sigma} g_{\sigma}$ where $f$ is the integrand of the integral to be minimized, the functions $\lambda_{\beta}$ are continuous multipliers, and $\mu_{\sigma}$ are constants. The generalization of the Lindeberg theorem which is 
obtained asserts, stated roughly, that if the Weierstrass condition in its strengthened form is satisfied along a curve $C_{0}$, then for every positive $\epsilon$ there exists a positive $\delta$ such that $J(C)>J\left(C_{0}\right)-\epsilon$ for every admissible curve $C$ whose length exceeds that of $C_{0}$ by at least $\delta$. By means of this theorem the author then obtains sufficient conditions and also generalizations of the theorem of Osgood (compare Bolza, loc. cit., p. 280). The results obtained by McShane in his dissertation (published in vol. 1 of these contributions) are used in an important way; his methods prove to be very useful for the problem dealt with in this paper.

The final paper primarily concerned with the Lagrange problem is no. 10, by Dr. Valentine (pp. 403-448). Its problem differs from the usual Lagrange problem in this respect, that the minimizing functions, in addition to being solutions of the differential equations $\psi_{\alpha}\left(x, y, y^{\prime}\right)=0$, must also satisfy a number of differential inequalities $\phi_{\beta}\left(x, y, y^{\prime}\right) \geqq 0$. The problem is treated by consideration of the equivalent problem, in which functions $y_{i}(x), z_{\beta}(x)$ are sought that satisfy the conditions of the given problem, except that the inequalities are replaced by the differential equations $\phi_{\beta}-z_{\beta}{ }^{2}=0$. By means of this equivalent problem, the necessary conditions of Euler, Weierstrass, and Clebsch are established. In the discussion of conjugate points, and of sufficient conditions, the author considers "arcs without corners composed of $n$ subarcs" such that $\phi_{1}=0$ on some of these, while the remaining functions of the set $\phi_{\beta}$ are positive on all the subarcs, without reference to the equivalent problem. Indeed this problem does not lend itself to the usual treatment because it is a singular problem, owing to the absence of the functions $z_{\beta}^{\prime}$ from the integrand. It may be possible to overcome this difficulty by use of the suggestions contained in a recent paper by Radon (Jahresbericht der Deutschen Mathematiker-Vereinigung, vol. 47 (1937), p. 220).

Dr. Denbow (pp. 449-484) treats a generalization of the Bolza problem obtained by replacing the end conditions by conditions involving a number of intermediate points as well, and by modifying all functions outside the integral sign in the functional that is to be minimized in a similar manner. It is then shown that this modified problem can be reduced to an equivalent Bolza problem in the usual form by the introduction of an enlarged set of unknown functions. By applying the methods for the treatment of a problem of Bolza as developed by Bliss, the usual necessary conditions and sufficient conditions for a minimum are obtained for normal arcs. It is pointed out that the method used in this problem can also be used to treat (1) the case in which the differential equations and the integrand in the functional that is to be minimized involve the coördinates of the endpoints and of a set of intermediate points; (2) a problem in which the functional to be minimized is of the form

$$
g\left(x_{1}, y_{i}\left(x_{1}\right), x_{0}, y_{i}\left(x_{0}\right), x_{2}, y_{i}\left(x_{2}\right)\right)+\int_{x_{1}}^{x_{0}} f_{1}\left(x, y, y^{\prime}\right) d x+\int_{x_{0}}^{x_{2}} f_{2}\left(x, y, y^{\prime}\right) d x,
$$

the finite side conditions have the arguments of $g$, and two sets of differential equations are to be satisfied, one on the interval $\left(x_{1}, x_{0}\right)$ and the other on the interval $\left(x_{0}, x_{2}\right)$ - problems of this sort have arisen in applications to economic theory; (3) a problem in which is sought the minimum of an integral in the class of curves with fixed endpoints and with a fixed finite number of finite discontinuities.

The main purpose of Dr. Smiley's paper (pp. 527-566) is to establish conditions which insure that an arc of class $D^{\prime}$ shall be a minimizing arc for the problem of Bolza in parametric form. In the proof of the theorem which accomplishes this purpose, the author follows the methods developed by Hestenes for the problem in ordinary form (see Transactions of this Society, vol. 42 (1937), p. 141), which in turn rest upon the definition of Mayer fields as given by Bliss. The necessary embedding theorem is obtained by the introduction of canonical variables. 
The paper by Dr. Cosby (pp. 53-84) is the only one in this collection which is concerned with double integrals. On the basis of Bliss' definition of a field of extremal surfaces, necessary and sufficient conditions are obtained that a two-parameter family of space curves $u(x, y, z)=a, v(x, y, z)=b$, be a transversal family. These conditions take the form of a pair of partial differential equations, one of the first order and one of the second, which constitute the analogue for the double integral of the Hamilton partial differential equation of the simple problem. The differential equations thus obtained can also be interpreted as a condition to be satisfied by functions $A(x, y, z), B(x, y, z)$, and $C(x, y, z)$ in order that the Hilbert invariant integral may be expressed by means of the line integral $\int A d x+B d y+C d z$. It is shown that, from the transversal families of an $n$-parameter family of fields $(n \geqq 2)$, an $(n+1)$-parameter family of extremal surfaces can be obtained.

Paper 3 (pp. 85-164) takes up a study of unilateral variations in the plane and in 3 -space. The literature on this problem is not very extensive; indeed, the second order conditions have thus far received little attention. Dr. Mancill makes a definite advance in the study of these conditions. He makes use of the geometric approach which has been useful in the simple problem. Families of extremaloids are introduced whose elements are obtained by piecing together extremaloids through each of the fixed endpoints and arcs tangent to the bounding curve, possibly partly coinciding with this curve. In terms of the behavior of the envelopes of these families, an analogue of the Jacobi necessary condition is formulated, following the method used by Graves (see American Journal of Mathematics, vol. 52 (1930), p. 1). Furthermore, conditions are stated which will insure the existence of fields of extremaloids, and by means of them sufficient conditions for a strong minimum are obtained for the case in which the bounding curve is not an extremal arc; a supplementary theorem deals with the special case in which this does happen. There is finally an extensive discussion of the case in which the bounding curve has corners. The problem of unilateral variations in space combines the results obtained by Graves with the methods used in the plane problem. The connection between the two problems is established by means of a curve $C$ on the bounding surface, which is the locus of the points at which the extremals of a certain one-parameter family touch the bounding surface $S$. An analogue of Jacobi's necessary condition, the existence of a field, and sufficient conditions are established. The bounding surface is supposed to be free from singularities.

The subject treated in Dr. Wiggin's paper (pp. 243-275) is the accessory boundary value problem for the problem of Bolza, associated with the study of the second variation in accordance with Bliss' method. By means of the condition $\int_{x_{1}}^{x_{2}} \eta_{i} \eta_{i} d x=1$, the variations of the minimizing functions and of the $x$-coördinates of the endpoints are normalized. The accessory minimum problem is put into the form of a problem of Bol$\mathrm{za}$, for which the Euler equations contain a parameter $\rho$; this in its turn is treated by means of an associated Lagrange problem with fixed endpoints. By use of the Jacobi condition for this problem the result is obtained that the second variation of the original problem will be non-negative if and only if the smallest characteristic value of $\rho$ is non-negative. A further result establishes the connection between the characteristic values of the boundary problem and the minimum value of the second variation in successive subclasses of admissible variations. The treatment follows in the main the methods familiar from the work of Richardson and others.

Dr. Bardell (pp. 277-312) deals with a problem of the calculus of variations in the large. He considers first the positive regular integral $\int_{x_{1}}^{x_{2}} F\left(x, y, x^{\prime}, y^{\prime}\right) d t$, satisfying the usual positive homogeneity condition on curves lying in a region $R$ of the $x y$-plane, extremal convex and bounded by a simple closed curve, non-singular and analytic 
except for a finite number of corners. Extremal arcs joining two fixed points 1 and 2 are called adjacent if there is no other extremal arc joining these same two points whose slope at 1 lies between the slopes of the given arcs at that point. The type of an extremal arc joining these two points is the number of points conjugate to 1 which lie between 1 and 2, in accordance with Morse's use of the term. A sequence $S_{k},(k=0,1, \cdots, n)$, of extremal arcs joining 1 and 2 is a set of adjacent arcs of type $k$, preceded and followed by arcs of type different from $k$. It is shown that adjacent sequences differ in type by unity if 2 is not on the envelope of the family of extremal arcs through 1 . The principal result is that, if $S_{k}$ represents the number of sequences of extremal arcs of type $k$ connecting two such points, then the relations $S_{i}>\sum_{k=-1}^{i-1}(-1)^{i+k-1} S_{k}$ for $i=0, \cdots, n-1$, and $S_{n}=\sum_{k=-1}^{n-1}(-1)^{n+k-1} S_{k}$, where $S_{-1}=1$, hold under the hypotheses mentioned above. It is shown furthermore that in the reversible case, in which $F\left(x, y, k x^{\prime}, k y^{\prime}\right)=|k| \cdot F\left(x, y, x^{\prime}, y^{\prime}\right)$, every sequence contains but a single arc, so that the relations just stated go over into a strengthened form of Morse's well known relations. These results are obtained by a careful study of the points of intersections of the extremal arcs through a fixed point.

The paper by Dr. Goldstine (pp. 313-358) lies in the field of the functional calculus. It considers a real-valued functional on the class $C$ of all real-valued functions $\xi$ on the interval $(0,1)$, on the subclass $D$ of $C$ determined by the condition $\int_{0}^{1} \xi(t) d t=0$, and on the subclass $N$ of $D$ whose elements have continuous derivatives on $(0,1)$. The first chapter establishes a representation of a continuous, bilinear, symmetric functional on the composite class $C_{1} C_{2}$, a necessary and sufficient condition that such a functional be non-negative on $D D$, and a necessary and sufficient condition that it be positive on $N N$. The second chapter, based on the paper of Hildebrandt and Graves (Transactions of this Society, vol. 29 (1927), p. 127), introduces definitions of first and second differentials of a real-valued functional on a Banach space, and shows that the second differential of such a functional is bilinear, continuous, and symmetric. The next chapter presents three necessary conditions and two sufficient conditions that a functional $J$ defined on a region $C_{0}$ in $C$ shall have a minimum at an element of one or another of certain subsets of $C_{0}$. These conditions are stated in terms of properties of the first and second differential of $J$ and correspond roughly to conditions familiar in the calculus of variations. For the purpose of generalizing the Jacobi condition a boundary value problem is introduced. An example is then given of a functional for which this boundary problem does not possess characteristic values, thus illustrating a deviation from the functionals of the calculus of variations. The last chapter deals with two applications of the general theory. The first of these is the simple problem of the calculus of variations with fixed endpoints, for which the condition for the positive character of the second variation is obtained in terms of the characteristic values of the associated boundary problem. The second is the problem of minimizing the integral $\int_{0}^{1} \int_{0}^{1} g\left[s, t, y(s), y^{\prime}(s)\right.$, $\left.y(t), y^{\prime}(t)\right] d s d t$ in the class of arcs from $\left(0, y_{1}\right)$ to $\left(1, y_{2}\right)$.

In paper 9 (pp, 359-402), Dr. Moscovitch discusses first the existence of Lagrange problems which have a given Hamiltonian function $H(x, y, z)$. It is shown that if $H$ is a function of class $C^{(4)}$, whose matrix $\left\|H_{z i z k}\right\|$ is identically of rank $n-m$ in a region $R$, and if there exists a curve $E, y_{i}=y_{i}(x), z_{i}=z_{i}(x)$, which satisfies the equations $y_{i}^{\prime}=H_{z i}$, then there exists a Lagrange problem which has $H$ as its Hamiltonian in the vicinity of $E$. Two Lagrange problems $f, \phi_{\alpha}$ and $g, \psi_{\alpha}$ are called equivalent if every solution of the equations $\phi_{\alpha}=0$ is a solution of $\psi_{\alpha}=0$ and conversely, and if $\int f d x$ and $\int g d x$ have the same value along every admissible curve. It is then shown that two Lagrange problems which have the same Hamiltonian are equivalent. The advance 
which these results make over the earlier work of Carathéodory consists in the replacement of the condition that a particular $m$-rowed determinant of the matrix $\left\|H_{z_{i} z_{k}}\right\|$ be different from 0 over the entire range of $x$ by the condition that this matrix be everywhere of rank $m$. The author then applies his results to the inverse problem for space curves. The determination of an integrand which yields a given four-parameter family of curves in 3-space as extremals can now be replaced by the determination of a Hamiltonian; this is reduced, by use of the Hamilton-Jacobi differential equation, to the determination of a function $W(x, y, z, b)$ such that the given extremals are solutions of the equations $W_{a}=c, W_{b}=d$. The conditions under which such a $W$ exists are not determined. Another approach to the solution of the inverse problem is made through its connection with contact transformations, as pointed out by Douglas (Transactions of this Society, vol. 29 (1927), p. 407). Here extremals and transversals are taken together and a relation is obtained, necessary and sufficient for the equivalence of a calculus of variations problem and a contact transformation, which extends a result of Douglas. Finally, the author obtains a condition that a family of curves in $n$-space and a given transversality relation be extremals and transversality for a calculus of variations problem.

The paper by Dr. Householder (no. 12, pp. 485-526) deals with the properties of focal points on extremals of a parametric problem in $n$-space transversal toa variety $V$ of $1,2, \cdots, n-1$ dimensions. This problem has been studied by Bliss for the plane (Transactions of this Society, vol. 3 (1902), p. 132) and by White for 3-space (ibid., vol. 13 (1912), p. 175). The results obtained by these authors suggest that, in case $V$ is a curve in $n$-space, the curvature of this curve will play an important part. Consequently the first section of the paper is devoted to the principal curvature of a curve in $n$-space, and its interpretation for a curve on a variety $V$, following the methods of Cartan in his treatment of Finsler spaces. These methods also underlie the discussion of the first and second variation of the integral $\int L\left(x, x^{\prime}\right) d t$, the conjugate point, and the Jacobi equations. The focal point of a curve $D$ is defined by an extension of the definition familiar from the simpler problems; the existence and position of focal points are discussed for varieties $V$ as well as for curves.

Anyone who looks through this book must be impressed by the active developments in the calculus of variations which are going forward at the University of Chicago. The volume under review shows that the modern theories are being cultivated as well as the problems and methods of the classical period.

ARNold DRESDEN 